\title{
Implosion and Heating Experiments of Fast Ignition Targets for FIREX-1 Project
}

H. Shiraga 1), J. Zhong 1), M. Koga 1), H. Nishimura 1), S. Fujioka 1), M. Tanabe 1), H. Azechi 1), T. Watari 1), H. Hosoda 1), K. Takeda 1), K. Otani 1), M. Nakai 1), K. Nagai 1), T. Norimatsu 1), N. Miyanaga 1), H. Nagatomo 1), T. Johzaki 1), K. Mima 1), A. Iwamoto 2), T. Mito 2), M. Isobe 2), and T. Ozaki 2)

1) Institute of Laser Engineering, Osaka Univ., 2-6 Yamada-Oka, Suita, Osaka, Japan

2) National Institute for Fusion Science, Oroshi-cho, Toki, Gifu, Japan

e-mail: shiraga@ile.osaka-u.ac.jp

\begin{abstract}
Implosion and heating experiments of Fast Ignition (FI) targets for FIREX-1 project have been performed with Gekko-XII and PW/LFEX lasers at the Institute of Laser Engineering, Osaka University. Typical FI target has a hollow cone for guiding the short-pulse heating laser beam at the time of the maximum compression. The cone is mounted so as to in one-side penetrate the shell target. Detailed implosion hydrodynamics, FI heating and core plasma formation of plastic (CD) shell target with gold cone have been clarified by observing those with fast imaging x-ray spectroscopy and neutron diagnostics. A new OneDimensional and Multi-channel Multi-Imaging X-Ray Streak Camera (1D+McMIXS) was developed for observation of time-resolved two-dimensional x-ray images and time-resolved two dimensional temperature distributions. Also, a monochromatic x-ray imager coupled with 2D-SIXS (Two-Dimensional Sampling Image $\mathrm{X}$-ray Streak camera) was developed for time resolved $\mathrm{x}$-ray line-emission imaging. Synchronization of the heating beam injection to the implosion dynamics has been monitored with an x-ray framing camera. With these instruments, one can observe heating properties of the imploded core such as spatial distribution of the heated region and its temporal evolution. It was found that the shape of the core is neither spherical nor uniform mainly because of the existence of the cone and moving toward the tip of the cone and interacting with it. Experimental results are compared with two-dimensional hydrodynamic simulations. Target design taking into account of these phenomena is quite important because such core movement and jet formation can affect the condition of the cone.
\end{abstract}

\section{Introduction}

Fast ignition is a new scheme for inertial confinement fusion, in which a high-power shortpulse laser is injected to the imploded core for heating the fuel core plasma [1-3]. A shell target with a cone for guiding the heating beam has been proposed for Fast Ignition scheme [4]. Implosion of such target is considered no longer symmetric nor uniform because of the existence of the cone. Furthermore, such asymmetric core may interact with the cone [5]. It is of great importance to observe hydrodynamics of imploded core plasma of such target, particularly in the viewpoint of symmetry, uniformity, and movement. Such core plasma become hot enough to emit a few to several $\mathrm{keV}$ x-rays during its short lifetime such as 100 ps. Thus ultrafast $\mathrm{x}$-ray imaging becomes an important diagnostic tool for measurement of the imploded core and its interaction with the cone.

An x-ray streak camera coupled with image sampling technique is one of solutions for such requirement. We have proposed and developed many types of those. MIXS (multi-imaging Xray streak camera) has been developed and successfully utilized in many implosion experiments [6-8]. Many types of spectroscopic application of MIXS have also been developed $[9,10]$. As another ultrafast two-dimensional x-ray image-sampling technique, 2DSIXS (two-dimensional sampling image x-ray streak camera) was developed, and proof-ofprinciple demonstration was performed [11]. We recently have improved the scheme and applied it for the first time to observation of the dynamics of imploded core plasma of a shell target with a cone for Fast Ignition $[12,13]$. 
Also we have developed a scheme for observing the synchronization of the heating beam injection to the imploded core plasma formation time by using a conventional gatedmicrochannel-based x-ray framing camera [14].

FIREX-1 project [15] is a program to perform implosion and heating experiments of Fast Ignition target with a cone by using Gekko-XII and PW/LFEX lasers at Institute of Laser Engineering, Osaka University. Implosion and heating of the fuel core plasma have been diagnosed with such ultrafast x-ray diagnostics as well as neutron diagnostics. Details of recent results are described in this article.

\section{Fast Imaging X-Ray Spectroscopy of the Imploded Core}

A new One-Dimensional (1D) and Multi-channel Multi-Imaging X-Ray Streak Camera (1D+McMIXS) [16] was developed to observe time-resolved two-dimensional x-ray images in multi spectral channels enabling us to derive time-resolved two dimensional (2D) temperature distributions of the core plasma with time- and special resolutions of 24 ps and $20 \mu \mathrm{m}$, respectively, as well as 1D images of the target to observe the implosion shell trajectory simultaneously. This enables us to determine the core formation time in the whole implosion process, and to compare those accurately with simulations. 1D+McMIXS system consisted of a multi-pinhole disk and an x-ray streak camera with three-slit photo cathode. 1D images of the target equator were made on the first cathode slit by using conventional imaging pinholes with different diameters and filters. Two sets of MIXS images were made on the second and the third slit cathodes by using two tilted pinhole arrays with different filters to obtain different spectral responses. Each pinhole array had 11 pinholes with a constant separation in between. After streaking the images, sequential 2D images were reconstructed by rearranging the time-sliced imagelets. Assuming a plasma model for x-ray emission, such as bremsstrahlung, one can derive plasma temperature from two spectral channels. Thus one can obtain time-resolved 2D temperature map of the imploded core plasma.

2D-SIXS (Two-Dimensional Sampling Image X-ray Streak camera) was improved and utilized for the first time in observation of the imploded core plasma of FI target with temporal and spatial resolutions of 24 ps and $20 \mu \mathrm{m}$, respectively [12]. Here we used two dimensionally distributed $40 \times 39$ pinholes to sample a 2D x-ray image on the cathode plane of an x-ray streak camera. Also, another 2D-SIXS, which was coupled with a high-spatialresolution monochromatic x-ray imager [17], was developed for time resolved x-ray lineemission images [13]. Two toroidally bent Bragg crystals (Quartz) were used for making spectrally selected monochromatic images at certain line emission spectra from the target material. By comparing the two line spectral images with assumption of appropriate plasma model, one can derive time-resolved 2D temperature and/or density maps.

Synchronization of the heating beam injection to the implosion dynamics has been monitored with an x-ray framing camera with gated micro channel plate driven by traveling voltage pulse by using the hard x-ray background due to hot electron generation by the heating beam as a signal representing the heating pulse [14]. Conventional framing images of the implosion were recorded with the same $\mathrm{x}$ ray framing camera except for the duration around the hard $\mathrm{x}$ ray signal. 
With these instruments, one can observe heating properties of the imploded core such as spatial distribution of the heated region and its temporal evolution.

\section{Imploded Core and Its Dynamics}

Implosion and heating experiments have been performed at Gekko-XII and PW lasers. Typical FI target has a hollow cone for guiding the short-pulse heating laser beam at the time of the maximum compression. Typical laser and target parameters were as follows. GekkoXII laser for implosion: $1.5-4.5 \mathrm{~kJ}$ in total $/ 1.5 \mathrm{~ns}$ pulse, 9 beams among 12 . PW laser for heating: up to $200 \mathrm{~J}$ in $1 \mathrm{ps}$ in 1 beam. CD shell: $500 \mu \mathrm{m}$ in diameter and $7 \mu \mathrm{m}$ in thickness, with a 10-20 $\mu \mathrm{m}$ thick Au cone of 30 degrees. The Au cone was coated with $10 \mu \mathrm{m}$ thick $\mathrm{CH}$ layer. A variety of parameters including $d$ (distance from the center to the cone tip), $\theta$ (opening angle of the cone), and $s$ (shift of the cone axis from the target center) was chosen.
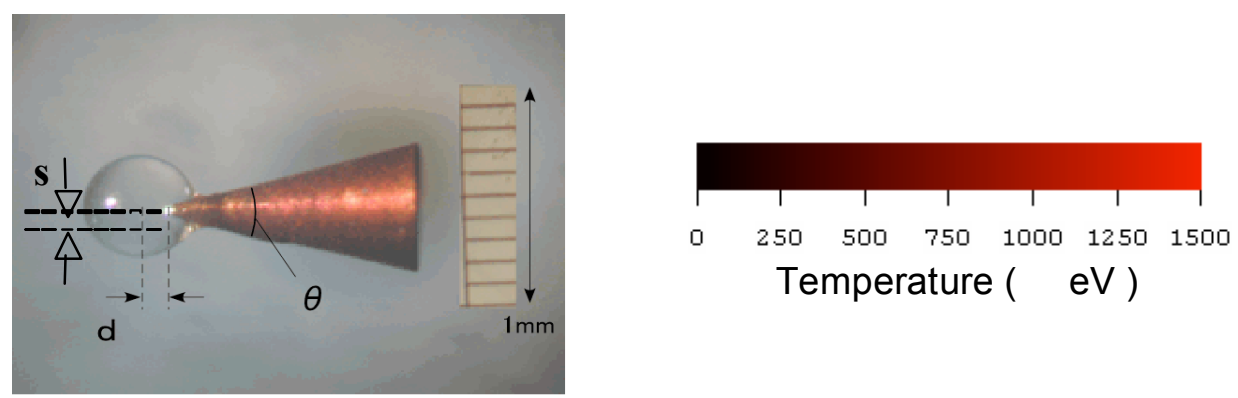

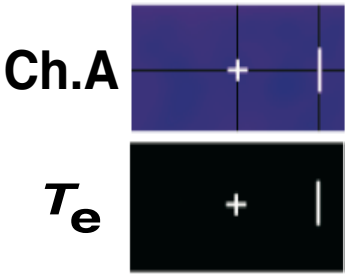

92

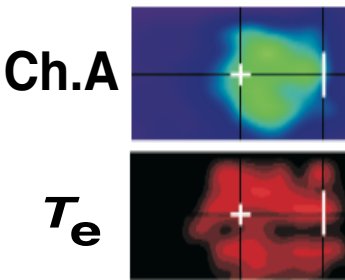

368

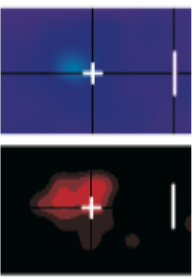

138

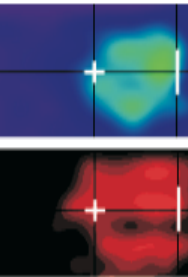

414

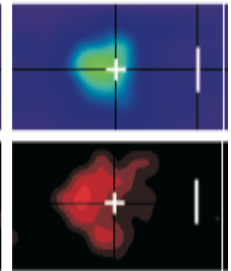

184

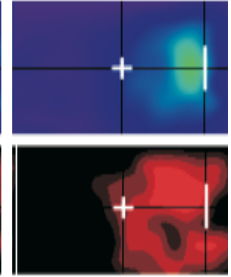

460

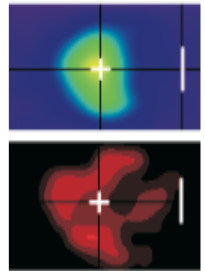

230

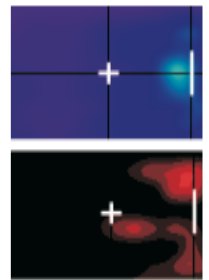

506

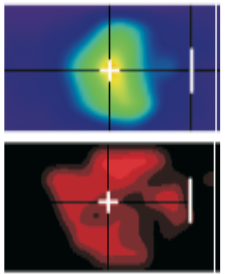

276

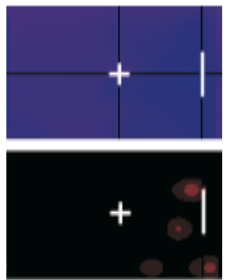

552

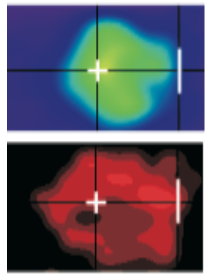

322

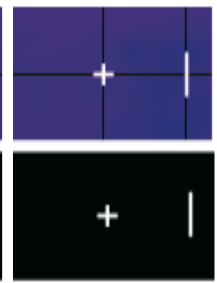

$598(\mathrm{ps})$

Time ( ps )

FIG. 1. Time-resolved x-ray images of channel A (2-5 keV) (upper) and temperature distribution (bottom) for three frames. Field of view of each frame is $120 \mu \mathrm{m}$ (vertical) $x 200 \mu \mathrm{m}$ (horizontal). White crosses and bars correspond to the initial positions of the center of the shell and the tip of the cone, respectively. Top left is a view of a plastic shell target with a Au cone.

Time-resolved core shape and its temperature distribution were successfully measured with $1 \mathrm{D}+\mathrm{McMIXS}$ as shown in Fig. 1 . In this case, $d=75 \mu \mathrm{m}, \theta=30 \mathrm{deg}$, and $s=0 \mu \mathrm{m}$, and there was no heating laser beam in this shot. Upper images at each time are x-ray emission images in spectral channel A of McMIXS. Channel A observed x-ray emission in a spectral range of 2-5 keV, while channel B (not shown in Fig.1) observed 2-3 keV. Electron temperature 
distribution was obtained from the signal intensity ratio of channel A and channel B as shown in the bottom of the images. Note that derived temperature is nominal because the x-ray emission spectrum was assumed to be bremsstrahlung, and radiation transport including absorption and reemission was not taken into account.

The X-ray emission from the core was found to start at the center of the target (Ch. A at $t=$ $184-230 \mathrm{ps}$ ), and then move toward the tip of the cone (Ch. A at $t=322-460 \mathrm{ps}$ ), and finally stop at the tip (Ch. A at $t=460-506 \mathrm{ps}$ ) and disappear (Ch. A at $t=506-598 \mathrm{ps}$ ). It was found that the shape of the core is neither spherical nor uniform mainly because of the existence of the cone (for example, Ch. A at $t=230 \mathrm{ps}$ ). A jet-like structure ejected from the nonuniform core toward the cone was observed (Ch. A at $t=276-322 \mathrm{ps}$ ). It is indicated that the temperature is high in the jet plasma near the cone $\left(T_{\mathrm{e}}\right.$ at $\left.t=230-276 \mathrm{ps}\right)$. Also the stagnating plasma at the tip of the cone is found to be hot ( $T_{\mathrm{e}}$ at $\left.t=322-460 \mathrm{ps}\right)$. These results indicate that the cone tin is first hit bv the iet and then the whole core plasma comes to the cone tip.

(a)
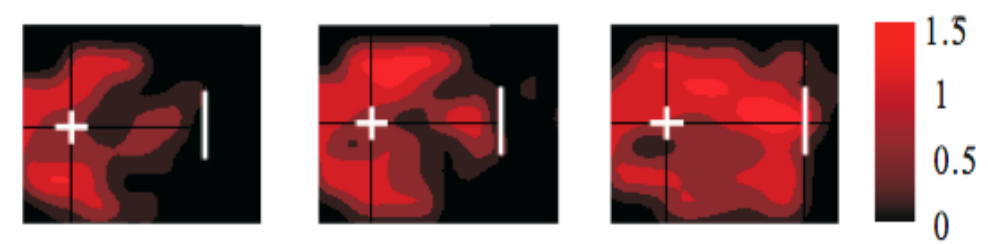

(b)
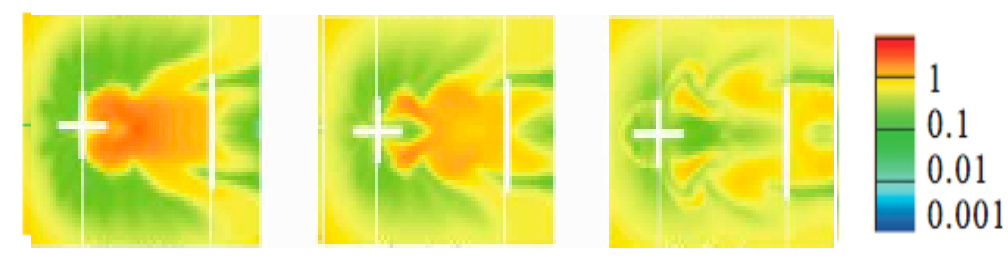

(c)

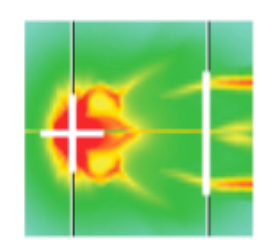

230 ps
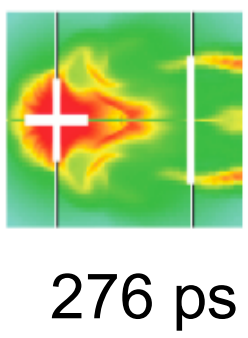
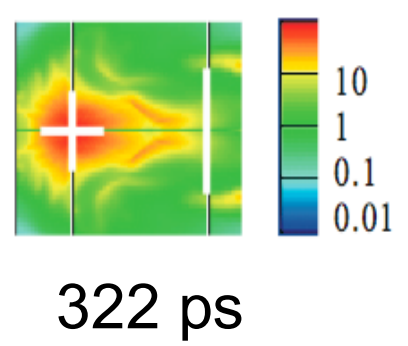

FIG. 2. Experimentally observed temperature (a), calculated temperature (b) and density (c) distributions of the core plasma and the jet. Temperatures are in $\mathrm{keV}$ and density in $\mathrm{g} / \mathrm{cm}^{3}$. Color scales are shown for each.

Comparison between experimentally observed temperature and results by $2 \mathrm{D}$ simulasion is shown in Fig. 2. 2D calculation was performed with PINOCO code [18]. Note that the experimental observation (a) is space-integrated along the line of sight and simulations $(b, c)$ are just cross sectional views. Both Fig. 2 (a) and (b) show that the central part of the core plasma is cool. Although the temperature is low, the density is high as shown in Fig. 2 (c), thus this part is bright in the emission image as shown in Fig. 1. It is found that the head (right hand) of the jet is hot, while the following part (left) of the jet is cold and dense (Fig. 2 (a) and (b)).

\section{Summary}

Hydrodynamics of the imploded core plasma of FI targets were investigated with ultrafast Xray spectroscopic imaging techniques. Experimental results are consistent with twodimensional hydrodynamic simulations in the viewpoint of the hydrodynamics of the core and 
its interaction with the cone. Target design taking into account of these phenomena is quite important because such core movement and jet formation can affect the condition of the cone at the time of the heating beam injection. Cone must be kept undisturbed when the heating laser is injected at the time of the maximum compression of the core

\section{References}

[1] T. Yamanaka (1983, unpublished).

[2] N. G. Basov, S. Yu. Gus'kov, and L. P. Feoktistov, J. Sov. Laser Res., 13, 396 (1992).

[3] M. Tabak, J. Hammer, M. E. Glinsky, W. L. Kruer, S. C. Wilks, J. Woodworth, E. M. Campbell, M. D. Perry, R. J. Mason, Phys Plasmaqs 1, 1626 (1994).

[4] R. Kodama, P. A. Norreys, K. Mima, A. E. Dangor, R. G. Evance, H. Fujita, Y. Kitagawa, K. Kurshelnick, T. Miyakoshi, N. Miyanaga, et al., Nature 412, 798 (2001); R. Kodama, H. Shiraga, K. Shigemori, Y. Toyama, S. Fujioka, H. Azechi, H. Fujita, H. Habara, T. Hall, Y. Izawa, et al., ibid, 418, 933 (2002).

[5] H. Shiraga, S. Fujioka, P. A. Jaanimagi, C. Stoeckl, R. B. Stephens, H. Nagatomo, K. A. Tanaka, R. Kodama, and H. Azechi, Rev. Sci Instrum. 75, 3921 (2004); R. Stephens, S. P. Hatchett, M. Tabak, C. Stoeckl, H. Shiraga, S. Fujioka, M. Bonino, A. Nikroo, R. Petrasso, T. C. Sangster, et al., Phys. Plasmas 12, 056312 (2005).

[6] H. Shiraga, M. Heya, A. Fujishima, O. Maegawa, K. Shimada, Y. Kato, T. Yamanaka, and S. Nakai, Rev. Sci Instrum. 66, 722 (1995).

[7] H. Shiraga, N. Miyanaga, M. Heya, M. Nakasuji. Y. Aoki, H. Azechi, T. Yamanaka and K. Mima, Rev. Sci Instrum. 68, 745 (1997).

[8] H. Shiraga, M. Heya, M. Nakasuji, N. Miyanaga, H. Azechi, H. Takabe, T. Yamanaka, and K. Mima, Rev. Sci Instrum. 68, 828 (1997).

[9] M. Heya, M. Nakasuji, H. Shiraga, N. Miyanaga, H. Azechi, H. Takabe, T. Yamanaka, and K. Mima, Rev. Sci Instrum. 68, 820 (1997).

[10] N. Miyanaga, Y. Aoki, H. Shiraga, K. Shimada, K. Fujimoto, M. Heya, and M. Nakasuji, Rev. Sci Instrum. 68, 817 (1997).

[11] H. Shiraga, M. Nakasuji, M. Heya, and N. Miyanaga, Rev. Sci. Instrum. 70, 620 (1999).

[12] H. Shiraga, M. Lee, N. Mahigashi, S. Fujioka, and H. Azechi (to be published in October, 2008 issue of Rev. Sci. Instrum.)

[13] M. Tanabe, T. Fujiwara, S. Fujioka, H. Nishimura, H. Shiraga, H. Azechi, and K. Mima (to be published in October, 2008 issue of Rev. Sci. Instrum.)

[14] M. Koga, T. Fujiwara, T. Sakaiya M. Lee, K. Shigemori, H. Shiraga, and H. Azechi (to be published in October, 2008 issue of Rev. Sci. Instrum.)

[15] H. Azechi, K. Mima, Y. Fujimoto, S. Fujioka, H. Homma, T. Jitsuno, T. Johzaki, M. Koga, J. Kawanaka, N. Miyanaga, M. Murakami, H. Nagatomo, K. Nagai, M. Nakai, T. Nakamura, K. Nishihara, H. Nishimura, T. Norimatsu, K. Shigemori, H. Shiraga, K. Tsubakimoto, A. Iwamoto, T. Mito, H. Sakagami, M. Isobe, T. Ozaki, O. Motojima, R. Kodama, K. Kondo, K.A. Tanaka, Y. Nakao, Y. Sentoku, A. Sunahara, T. Taguchi, in this proceedings, $\mathrm{OV} / 4-1$.

[16] J. Zhong, H. Shiraga, and H. Azechi (to be published in October, 2008 issue of Rev. Sci. Instrum.)

[17] K. Fujita, H. Nishimura, I. Niki, J. Funakura, I. Uschmann, R. Butzbach, and E. Förster, M. Nakai, M. Fukao, A. Sunahara, H. Takabe, and T. Yamanaka, Rev. Sci. Instrum. 72, 744 (2001).

[18] H. Nagatomo, T. Johzaki, T. Nakamra, H. Sakagami, A. Sunahara, and K. Mima, Phys. Plasma 14, 056303 (2007). 